\title{
Exact Outage Probability Analysis for Relay-aided Underlay Cognitive Communications
}

\author{
Zakaria El-Moutaouakkil ${ }^{(1,2)}$, Kamel Tourki ${ }^{(1)}$, Khalid A. Qaraqe ${ }^{(1)}$, Samir Saoudi $^{(2)}$ \\ (1) Electrical \& Computer Engineering Program, Texas A\&M University at Qatar, Doha, Qatar \\ ${ }^{(2)}$ Institut TELECOM/TELECOM Bretagne/UMR CNRS 3192 Lab-STICC, Brest, France \\ Emails: \{kamel.tourki, khalid.qaraqe\}@qatar.tamu.edu, \{zakaria.elmoutaouakkil, samir.saoudi\}@ telecom-bretagne.eu
}

\begin{abstract}
In a spectrum sharing underlay context, we investigate the exact derivation of the outage probability for relay-aided cognitive radio communications. To give more degrees of freedom to the secondary system in acquiring a targeted quality-of-service (QoS) under the primary system interference constraint, the secondary link is assisted by a set of relays acting in a two-hop decode-and-forward selective relaying mode. By means of the cumulative distribution functions of the received signal-to-noise ratio (SNR) at the secondary receiver, we derive the end-to-end outage probability of the secondary system in its closed form. The analytical and simulation results are then compared and interestingly shown to perfectly match over the entire interference threshold region.
\end{abstract}

Index Terms-Outage probability, cognitive radio, spectrum sharing, underlay, QoS, primary system, secondary system.

\section{INTRODUCTION}

In response to the ever-growing stress put on the wireless spectrum medium, cognitive radio (CR) has recently been emerged as a new principle to cope with the under-utilization of the wireless spectrum; thereby enabling more wireless-end users to acquire with more flexibility the desired quality-of-service (QoS). Many primordial concepts have been inherited from the original idea of cognitive radio, drawing three active research lines [1] under the names of spectrum interweave, spectrum overlay, and spectrum underlay. In this contribution, we focus on the underlay spectrum sharing concept as a way to allow secondary (unlicensed) users (SUs) to share the same licensed spectrum band with the primary users (PUs). However, as far as the interference issue is concerned, the secondary user's transmit power must be kept under a certain threshold largely determined by the primary user. Therefore, the SUs have the opportunity to use the licensed spectrum alongside with the PUs as long as the SUs respect the interference constraint.

To maintain a reasonable QoS for the SU even though the interference constraint may not be favorable, selective cooperative relaying has recently been incorporated into cognitive radio systems to achieve a desirable secondary system outage performance [2]-[7]. Indeed, we differentiate between two relay selection criterions depending on which relaying protocol is being adopted. For instance, the best harmonic mean of the first and second

This publication was made possible by NPRP grant 08-101-2-025 from the Qatar National Research Fund (a member of Qatar Foundation). The statements made herein are solely the responsibility of the authors. hops channel gains can be used [8] for amplify-and-forward (AF) relaying, while the minimum of the two hops channel gains (max-min criterion) can be used [9] for decode-and-forward (DF) relaying. In [3], the authors considered a relay selection based on the max-min criterion in the absence of the direct secondary link, and accordingly the outage probability was derived. However, the direct secondary link was considered in [4], but only a tight lower bound on the outage probability was investigated. In [10], the authors focused on a cognitive relay network under PU's outage constraint, where the SU's transmission should not affect the PU's transmission and maintain the outage probability at the primary receiver under a predetermined value. However, and considering that direct secondary link may or may not be used, they derived the outage probability of SU based on an upper bound expression of the PU's outage probability, and the number of involved relays is determined by exhaustive comparisons. Under several relay selection strategies, the authors in [5] carried out an asymptotic outage analysis for the secondary system, whereas the exact formula of the outage probability is derived in [6] by relaxing the maximum transmit power constraint and reducing the number of active relays to one. Both work in [5] and [6] did not consider the direct link between the secondary transmitter and receiver. Very recently, the authors in [11] investigated the exact outage analysis of the secondary system under limitted transmit power constraints and best second-hop DF relay selection. However, the authors did not consider the particular case when possibly no relay succeeds to meet its SNR threashold during the first-hop, which impacts the derivation of the end-to-end outage probability.

In addressing the SU transmission reliability limited by the interference constraint while targeting the above restrictions in previous works, we derive an exact analysis of the end-to-end outage probability for relay-aided secondary system transmissions. Among many relay candidates, only one relay will be selected to assist the secondary transmitter in its transmission towards the secondary receiver. The relay selection follows from satisfying two conditions: first, the relay must succeed in meeting its SNR threshold (thus, not falling in outage) during the first-hop, and second, lead to the maximum second-hop received SNR. Unlike the max-min criterion applied on the two hops channel gains, the proposed relay selection scheme is optimal in the sense of capturing the effect of the interference channel between the selected 
relay and the primary receiver. Also, the similarity between the relay selection scheme and the implemented DF relaying protocol remains a crucial fact in deriving accurate outage analysis. In this paper, our contributions can be summarized in three points:

- We provide closed form expressions of the cumulative distribution functions of the received SNR at the level of the relay and secondary receiver during both hops;

- The end-to-end outage probability is then given in its exact expression that is independent of the system's geometry and settings, in particular the number of assisting relays;

- Through simulation results, we investigate the optimal relay location in between the secondary transmitter and receiver that leads to the optimal outage performance.

\section{SyStem Model}

As illustrated in Fig. 1, our cognitive radio setup consists of a secondary (unlicensed) single-antenna transmitter-receiver pair $\left(S_{\mathrm{T}}, S_{\mathrm{R}}\right)$ that shares the same spectrum band with a primary (licensed) single-antenna transmitter-receiver pair $\left(P_{\mathrm{T}}, P_{\mathrm{R}}\right)$. For the purpose of reliability-efficient secondary system transmissions, a cluster $\mathcal{C}$ of $\mathrm{K}$ relays $\mathcal{C}=\left\{R_{\mathrm{k}}: \mathrm{k}=1, \ldots, \mathrm{K}\right\}$ operating in a half-duplex decode-and-forward selective relaying mode is supposed to assist $S_{\mathrm{T}}$ in its transmission towards $S_{\mathrm{R}}$. The interference channel gains $h_{\mathrm{sp}}$ and $h_{\mathrm{kp}}$ for $\mathrm{k} \in\{1, \ldots, \mathrm{K}\}$ correspond to the links connecting $S_{\mathrm{T}}$ and $R_{\mathrm{k}}$ to the primary receiver $P_{\mathrm{R}}$. Both are assumed to be drawn from a Rayleigh distribution with variances $\lambda_{\mathrm{sp}}$ and $\lambda_{\mathrm{kp}}$, respectively. Also, the channel gains $h_{\mathrm{sk}}$ (between $S_{\mathrm{T}}$ and $R_{\mathrm{k}}$ ), $h_{\mathrm{ks}}$ (between $R_{\mathrm{k}}$ and $S_{\mathrm{R}}$ ), $h_{\mathrm{ss}}$ (between $S_{\mathrm{T}}$ and $S_{\mathrm{R}}$ ) and $h_{\mathrm{pp}}$ (between $P_{\mathrm{T}}$ and $P_{\mathrm{R}}$ ) are all assumed to be Rayleigh distributed with variances $\lambda_{\mathrm{sk}}, \lambda_{\mathrm{ks}}, \lambda_{\mathrm{ss}}$ and $\lambda_{\mathrm{pp}}$, respectively. Pertaining to the underlay principle, and prior to each secondary transmission either from $S_{\mathrm{T}}$ or $R_{\mathrm{k}}$ towards $S_{\mathrm{R}}$, both nodes must adhere to the following interference constraint,

$$
Q \leq \mathrm{I},
$$

where $Q$ stands for the interference that $S_{\mathrm{T}}$ or $R_{\mathrm{k}}$ may induce at the primary receiver $P_{\mathrm{R}}$ when transmitting simultaneously with $P_{\mathrm{T}}$, while I corresponds to the maximum amount of interference that $P_{\mathrm{R}}$ can tolerate. In fact, $S_{\mathrm{T}}$ must keep its transmit power per symbol duration under the maximum,

$$
\mathrm{P}=\frac{\mathrm{I} N_{0}}{\left|h_{\mathrm{sp}}\right|^{2}},
$$

so as not to violate (1). $N_{0}$ denotes the variance of the additive noise at the primary receiver $P_{\mathrm{R}}$ and at the secondary receiver $S_{\mathrm{R}}$ as well and is modeled as a zero-mean circular symmetric complex Gaussian variable. Furthermore, $\left|h_{\mathrm{sp}}\right|^{2}$ corresponds to the squared module $|.|^{2}$ of the interference channel gain $h_{\mathrm{sp}}$ between $S_{\mathrm{T}}$ and $P_{\mathrm{R}}$. The proposed ruling on the best relay selection criterion is given by,

$$
\mathrm{r}=\underset{\mathrm{k} \mid R_{\mathrm{k}} \in C}{\arg \max }\left\{\frac{\mathrm{I}\left|h_{\mathrm{ks}}\right|^{2}}{\left|h_{\mathrm{kp}}\right|^{2}}\right\},
$$

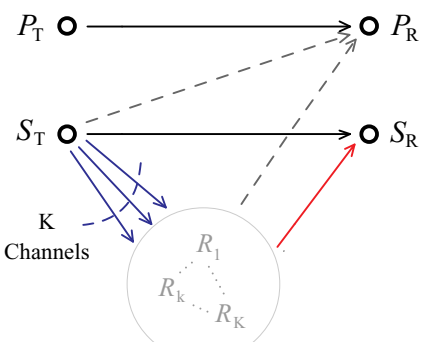

Cluster of K relays

Figure 1. Relay-aided Cognitive Radio System Model.

where $C$ is a subset within $\mathcal{C}$ including all the relays that do not fall in the outage during the first-hop, thus its cardinal $|C|$ may be equal to $\mathrm{i} \in\{0, \ldots, \mathrm{K}\}$. Eventually, the communication scheme between $S_{\mathrm{T}}$ and $S_{\mathrm{R}}$ spans two hops. During the first-hop, $S_{\mathrm{R}}$ receives the transmitted signal from $S_{\mathrm{T}}$, while during the secondhop, $S_{\mathrm{R}}$ receives from $R_{\mathrm{r}}$ what it successfully decoded during the first-hop. In the particular case of $|C|=0$, the secondary system will stand idle during the second-hop. However, this latter situation may rarely occur for a moderate number of relays. Thus, at the end of the second-hop, the receiver $S_{\mathrm{R}}$ may sum up the two instantaneous received SNRs during both hops $\gamma_{\mathrm{ss}}$ and $\gamma_{\mathrm{rs}}$, respectively;

$$
\gamma_{\mathrm{ss}}+\gamma_{\mathrm{rs}}=\mathrm{I}\left(\frac{\left|h_{\mathrm{ss}}\right|^{2}}{\left|h_{\mathrm{sp}}\right|^{2}}+\frac{\left|h_{\mathrm{rs}}\right|^{2}}{\left|h_{\mathrm{rp}}\right|^{2}}\right) .
$$

The conditional cumulative distribution function (CDF) of the instantaneous received SNR at the receiver $S_{\mathrm{R}}$ during the firsthop can be expressed as

$$
F_{\left.\gamma_{\mathrm{ss}}|| h_{\mathrm{sp}}\right|^{2}=z}(x)=1-e^{-\frac{x z}{1 \lambda_{\mathrm{ss}}}}
$$

Since the relays are forming among each other a cluster of $\mathrm{K}$ relays, we can in fact assume that $\lambda_{\mathrm{sr}_{\mathrm{k}}}=\lambda_{\mathrm{sr}}, \lambda_{\mathrm{r}_{\mathrm{k}} \mathrm{p}}=\lambda_{\mathrm{rp}}$ and $\lambda_{\mathrm{r}_{\mathrm{k}} \mathrm{S}}=\lambda_{\mathrm{rs}} \forall \mathrm{k} \in\{1, \ldots, \mathrm{K}\}$. Thus, similar to (5), the conditional CDF of $\gamma_{\mathrm{sr}_{\mathrm{k}}}$ (defined as the instantaneous received SNR at node $\mathcal{R}_{\mathrm{k}}$ during the first-hop) can also be expressed as

$$
F_{\left.\gamma_{\mathrm{sk}}|| h_{\mathrm{sp}}\right|^{2}=z}\left(y_{\mathrm{k}}\right)=1-e^{-\frac{y_{\mathrm{k}} z}{1 \mathbf{\lambda}_{\mathrm{sr}}}} .
$$

Let us now consider that $\mathrm{i} \in\{1, \ldots, \mathrm{K}\}$ is the cardinal of the subset $C$, i.e., i relays are now eligible to forward the decoded signal within the second-hop. According to the proposed best relay selection method (3), the conditional $\mathrm{CDF}$ of the received instantaneous SNR $\gamma_{\mathrm{rs}}$ at the level of node $\mathcal{D}_{\mathrm{s}}$ during the secondhop is given by

$$
\begin{aligned}
F_{\gamma_{\mathrm{rs}|| C \mid=\mathrm{i}}}(y) & =P_{r}\left[\gamma_{\mathrm{rs}} \leq y|| C \mid=\mathrm{i}\right] \\
& =P_{r}\left[\max \left\{\frac{\mathrm{I}\left|h_{\mathrm{ks}}\right|^{2}}{\left|h_{\mathrm{kp}}\right|^{2}}\right\} \leq y \mid R_{\mathrm{k}} \in C\right] .
\end{aligned}
$$

Irrespective of the relays indices belonging to the subset $C$, we will clearly see in the following that $F_{\gamma_{\mathrm{rs}|| C \mid=\mathrm{i}}}(y)$ does not depend on 
the relay indices within $C$, rather it only depends on the cardinal $|C|=\mathrm{i}$. Consequently, we can, without loss of generality, consider for the sake of simplicity that $C=\left\{\mathcal{R}_{1} \ldots, \mathcal{R}_{\mathrm{i}-1}, \mathcal{R}_{\mathrm{i}}\right\}$. Equation (7) can then be re-expressed as

$$
\begin{aligned}
F_{\gamma_{\mathrm{r}|| G \mid=\mathrm{i}}}(y) & =\prod_{\mathrm{j}=0}^{\mathrm{i}} P_{r}\left[\frac{\mathrm{I}\left|h_{\mathrm{js}}\right|^{2}}{\left|h_{\mathrm{jp}}\right|^{2}} \leq y\right] \\
& =\left[1-\frac{1}{1+\frac{y \lambda_{\mathrm{rp}}}{\mathrm{I} \lambda_{\mathrm{rs}}}}\right]^{\mathrm{i}}=\sum_{\mathrm{j}=0}^{\mathrm{i}}\left(\begin{array}{l}
\mathrm{i} \\
\mathrm{j}
\end{array}\right) \frac{(-1)^{\mathrm{j}}}{\left(1+\frac{y \lambda_{\mathrm{rp}}}{\mathrm{I} \lambda_{\mathrm{rs}}}\right)^{\mathrm{j}}}
\end{aligned}
$$

\section{Outage Probability Analysis}

The end-to-end outage probability of the proposed two-hop cognitive radio communication system can be defined as the sum of two probabilities,

$$
P_{\text {out }}(\mathrm{I}, \Phi, \mathrm{K})=P_{\text {out }}^{1}(\mathrm{I}, \Phi, \mathrm{K})+\sum_{\mathrm{i}=1}^{\mathrm{K}}\left(\begin{array}{c}
\mathrm{K} \\
\mathrm{i}
\end{array}\right) P_{\text {out }}^{2}(\mathrm{I}, \Phi, \mathrm{i}, \mathrm{K}),
$$

where $P_{\text {out }}^{1}(\mathrm{I}, \Phi, \mathrm{K})$ describes the event that $\gamma_{\mathrm{ss}}<\Phi$ and $\gamma_{\mathrm{sk}}<$ $\Phi \forall \mathrm{k} \in\{1, \ldots, \mathrm{K}\}$ with $\Phi=2^{2 \mathrm{R}}-1$ and $\mathrm{R}$ is the transmission rate, while $P_{\text {out }}^{2}(\mathrm{I}, \Phi, \mathrm{i}, \mathrm{K})$ corresponds to the event when $|C|=$ $\mathrm{i} \geq 1$, yet the sum $\gamma_{\mathrm{ss}}+\gamma_{\mathrm{rs}}$ of the received SNRs at $S_{R}$ during the first and second hops is still inferior to $\Phi$. The first probability $P_{\text {out }}^{1}(\mathrm{I}, \Phi, \mathrm{K})$ is given by (10) which, using equations (5) and (6), can further be expressed as (11). As for the second probability $P_{\text {out }}^{2}(\mathrm{I}, \Phi, \mathrm{i}, \mathrm{K})$, its exact derivation (the reader may kindly refer to the Appendix for more details) is given by (13), where

$$
\begin{aligned}
B & =1+\frac{\Phi \lambda_{\mathrm{sp}}}{\mathrm{I} \lambda_{\mathrm{ss}}}+\frac{(\mathrm{i}+\mathrm{m}) \Phi \lambda_{\mathrm{sp}}}{\mathrm{I} \lambda_{\mathrm{sr}}} \\
C & =\frac{\lambda_{\mathrm{sp}}}{\mathrm{I} \lambda_{\mathrm{ss}}} \\
D & =\frac{\lambda_{\mathrm{rp}}}{\mathrm{I} \lambda_{\mathrm{rs}}} .
\end{aligned}
$$

Finally, substituting the closed forms of (11) and (13) in (9) will lead to a closed form of the end-to-end outage probability. It is worth noting here that, unlike the outage analysis carried out in [4] where a lower bound on the outage probability is derived under the assumptions of symmetric secondary network geometry and small number $\mathrm{K}$, the derived closed form of the end-to-end outage probability is valid for any value $K$ and channel statistics $\lambda_{\mathrm{ss}}, \lambda_{\mathrm{sr}}$, $\lambda_{\mathrm{sr}}$ and $\lambda_{\mathrm{pp}}$. Most importantly, it provides useful insights into the impact of the proposed selective relaying scheme on the secondary system transmission reliability. For comparison purposes, the lower bound in [4] is reported in (14) in its general expression.

\section{Simulations Results}

\section{A. Network Geometry}

When taking into account large-scale variations of the cognitive radio channel, the second-order statistics of the link connecting each pair of transmitter-receiver within our system can be expressed as $\lambda_{\mathrm{ss}}=d_{\mathrm{ss}}^{-\nu}, \lambda_{\mathrm{sr}}=d_{\mathrm{sr}}^{-\nu}, \lambda_{\mathrm{rs}}=d_{\mathrm{rs}}^{-\nu}$ and $\lambda_{\mathrm{sp}}=d_{\mathrm{sp}}^{-\nu}$;

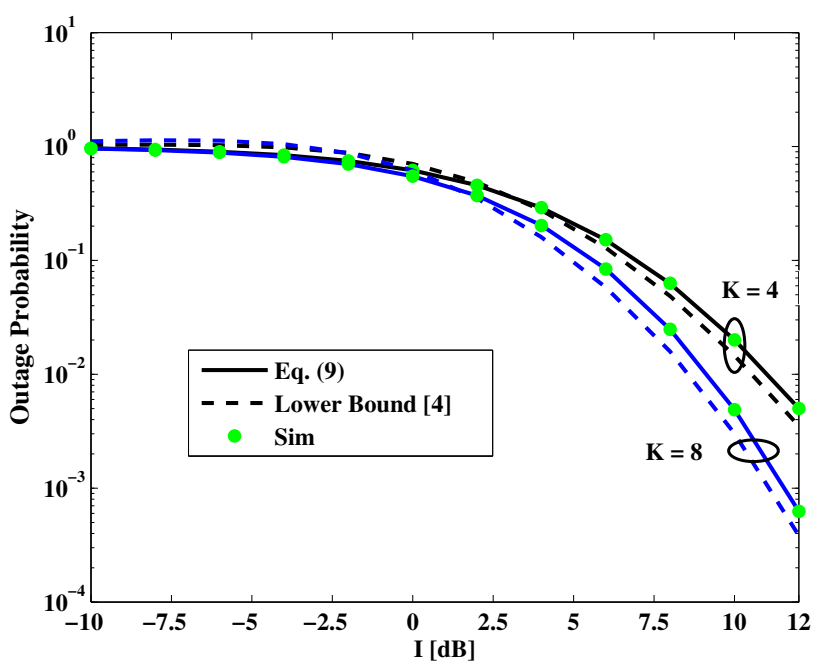

Figure 2. Outage Probability vs Interference threshold for $d_{\mathrm{sr}}=d_{\mathrm{rs}}=1$.

depending on the local distances ${ }^{1} d_{\mathrm{ss}}$ (between $S_{\mathrm{T}}$ and $S_{\mathrm{R}}$ ), $d_{\mathrm{sr}}$ (between $S_{\mathrm{T}}$ and $R_{\mathrm{k}}$ ), $d_{\mathrm{rs}}$ (between $R_{\mathrm{k}}$ and $S_{\mathrm{R}}$ ) and $d_{\mathrm{sp}}$ (between $S_{\mathrm{T}}$ and $P_{\mathrm{R}}$ ), respectively. The parameter $\nu$ corresponds to the path-loss exponent whose value is set to 4 as a practical value. For the sake of simplicity, we consider, without loss of generality, a normalized configuration in which the cluster $\mathcal{C}$ is located between the secondary transmitter $S_{\mathrm{T}}$ and the secondary receiver $S_{\mathrm{R}}$ such that $d_{\mathrm{sr}}+d_{\mathrm{rs}}=d_{\mathrm{ss}}=d_{\mathrm{sp}}=1$.

\section{B. Simulation Results}

In this section, we compare the analytical and simulation results of the end-to-end outage probability for different secondary system settings. For benchmarking purposes, these results will also be compared to the lower bound given in [4] and reported in its general expression in (14). In the case of a symmetric secondary system geometry, it is quiet remarkable from Fig. (2) and (3) that both the derived closed form of the outage probability and its simulated value perfectly coincide over the entire interference threshold region, while the lower bound is seen to be under their curves. However, for values inferior to $\mathrm{I}=0 \mathrm{~dB}$ in the case of $d_{\mathrm{sr}}=d_{\mathrm{rs}}=1$ and $\mathrm{I}=-4 \mathrm{~dB}$ in the case of $d_{\mathrm{sr}}=d_{\mathrm{rs}}=0.5$, the curve of the lower bound crosses the curve of the outage probability and goes up to attain values greater than 1 . We deduce then that the lower bound in [4] is valid only for moderate to high values (typically greater than $0 \mathrm{~dB}$ ) of the interference threshold I. In Fig. (4), the closed form of the outage probability is displayed in a $3 \mathrm{D}$ basis as a function of the interference threshold and the distance between the secondary transmitter and the cluster $\mathcal{C}$. The

\footnotetext{
${ }^{1}$ Since the assisting relays are forming among each other a cluster, the distance between two relays is assumed negligible compared to the distance between these relays and either the transmitter or receiver of each of the two primary and secondary systems. Therefore, all the relays are supposed to be located inside a cluster whose center is regraded as the position of each relay within it.
} 


$$
\begin{aligned}
& P_{\text {out }}^{1}(\mathrm{I}, \phi, \mathrm{K})=P_{r}\left[\gamma_{\mathrm{ss}}<\Phi, \gamma_{\mathrm{s} 1}<\Phi, \ldots, \gamma_{\mathrm{sK}}<\Phi\right] \\
& =\int_{0}^{+\infty} P_{r}\left[\gamma_{\mathrm{ss}}<\Phi, \gamma_{\mathrm{s} 1}<\Phi, \ldots, \gamma_{\mathrm{sK}}<\Phi \mid z\right] f_{\left|h_{\mathrm{sp}}\right|^{2}}(z) d z \\
& =\sum_{\mathrm{j}=0}^{\mathrm{K}}\left(\begin{array}{c}
\mathrm{K} \\
\mathrm{j}
\end{array}\right)(-1)^{\mathrm{j}}\left[\frac{1}{1+\frac{\mathrm{j} \Phi \lambda_{\mathrm{sp}}}{\mathrm{I} \lambda_{\mathrm{sr}}}}-\frac{1}{1+\frac{\mathrm{j} \Phi \lambda_{\mathrm{sp}}}{\mathrm{I} \lambda_{\mathrm{sr}}}+\frac{\Phi \lambda_{\mathrm{sp}}}{\mathrm{I} \lambda_{\mathrm{ss}}}}\right] \\
& P_{\text {out }}^{2}(\mathrm{I}, \Phi, \mathrm{i}, \mathrm{K})=P_{r}\left[\gamma_{\mathrm{ss}}+\gamma_{\mathrm{rs}}<\Phi, \gamma_{\mathrm{s} 1} \geq \Phi, \ldots, \gamma_{\mathrm{si}} \geq \Phi, \gamma_{\mathrm{si}+1}<\Phi, \ldots, \gamma_{\mathrm{sK}}<\Phi\right] \\
& =\sum_{\mathrm{m}=0}^{\mathrm{K}-\mathrm{i}}\left(\begin{array}{c}
\mathrm{K}-\mathrm{i} \\
\mathrm{m}
\end{array}\right)(-1)^{\mathrm{m}}\left[\sum_{\mathrm{c}=0}^{\mathrm{i}}\left(\begin{array}{l}
\mathrm{i} \\
\mathrm{c}
\end{array}\right) \frac{(-1)^{\mathrm{c}}}{\left(\frac{\mathrm{i}+\mathrm{m}) \Phi \lambda_{\mathrm{sp}}}{\mathrm{I} \lambda_{\mathrm{sr}}}+1\right)(1+\Phi D)^{\mathrm{c}}}-\left\{\sum_{\mathrm{d}=0}^{\mathrm{i}}\left(\begin{array}{l}
\mathrm{i} \\
\mathrm{d}
\end{array}\right) \frac{(-1)^{\mathrm{d}}}{(B-C \Phi)(1+\Phi D)^{\mathrm{d}}}\right.\right. \\
& \left.\left.+\sum_{\mathrm{n}=0}^{\mathrm{i}}\left(\begin{array}{l}
\mathrm{i} \\
\mathrm{n}
\end{array}\right)(-1)^{\mathrm{n}} \frac{C^{\mathrm{n}}}{(\mathrm{n}+1) D^{\mathrm{n}}}\left(\frac{{ }_{2} \mathrm{~F}_{1}\left(\mathrm{n}, \mathrm{n}+1, \mathrm{n}+2, \frac{C+B D}{D(B-C \Phi)}\right)}{(C \Phi-B)^{\mathrm{n}+1}}-\frac{{ }_{2} \mathrm{~F}_{1}\left(\mathrm{n}, \mathrm{n}+1, \mathrm{n}+2, \frac{C+B D}{B D}\right)}{(-1)^{\mathrm{n}+1} B^{\mathrm{n}+1}}\right)\right\}\right] \\
& P_{\text {out }}^{L}(I, \phi, K)=P_{\text {out }}^{1}(\mathrm{I}, \Phi, \mathrm{K})+\sum_{\mathrm{i}=1}^{\mathrm{K}}\left(\begin{array}{c}
\mathrm{K} \\
\mathrm{i}
\end{array}\right) \frac{\left(\lambda_{\mathrm{sp}} \Phi\right)^{\mathrm{K}+1}}{\left(\lambda_{\mathrm{sp}} \Phi+2 \lambda_{\mathrm{ss}} \mathrm{I}\right)\left(\lambda_{\mathrm{sp}} \Phi+2 \lambda_{\mathrm{rs}} \mathrm{I}\right)^{\mathrm{i}}}\left(\frac{\lambda_{\mathrm{sr}} \mathrm{I}}{\lambda_{\mathrm{sp}} \Phi+\lambda_{\mathrm{sr}} \mathrm{I}}\right)^{\mathrm{i}}\left(\frac{\lambda_{\mathrm{sp}} \mathrm{I}}{\lambda_{\mathrm{sp}} \Phi+\lambda_{\mathrm{sr}} \mathrm{I}}\right)^{\mathrm{K}-\mathrm{i}}
\end{aligned}
$$

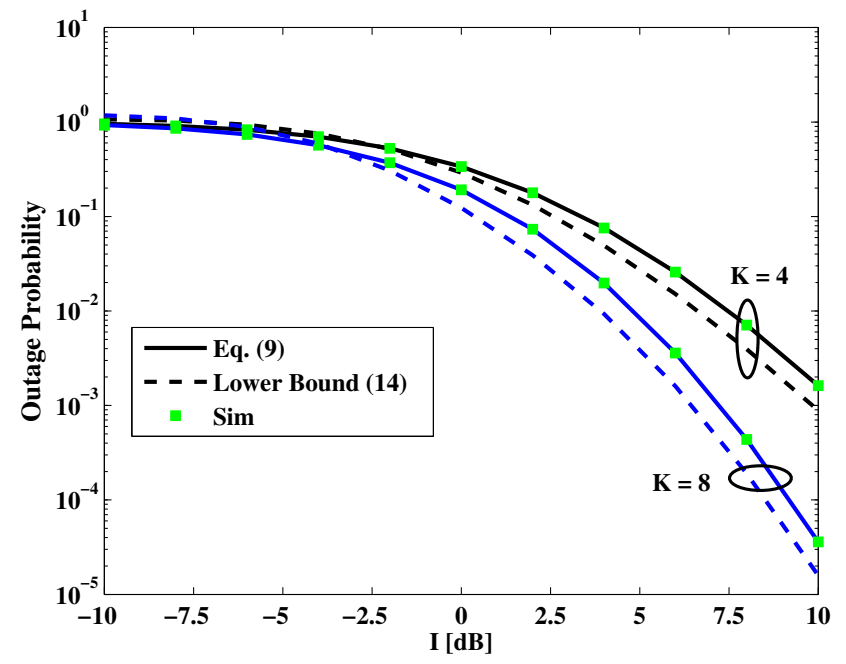

Figure 3. Outage Probability vs Interference threshold for $d_{\mathrm{sr}}=d_{\mathrm{rs}}=0.5$.

optimal value for $d_{\mathrm{sr}}$ leading to the best outage performance for $\mathrm{K}=4$ is found to be around $d_{\mathrm{opt}}=d_{\mathrm{sr}}=0.4$ which is quiet different from the conventional value $d_{\mathrm{sr}}=0.5$. Both values $d_{\mathrm{sr}}=0.5$ and $d_{\mathrm{opt}}$ are considered in Fig. (5) to investigate the impact of the number of relays $\mathrm{K}$ on the overall secondary system outage performance. It is observed that with a number $\mathrm{K}=8$, our secondary system can resist to a decrease of about $3.5 \mathrm{~dB}$ in the interference threshold to maintain the same outage performance $10^{-3}$ achieved when $K=4$. This suggests that with an increased number of relays, the secondary link becomes more insensitive to the primary system interference constraint. The great impact of the relays is also observed in Fig. (5) when we compare the outage

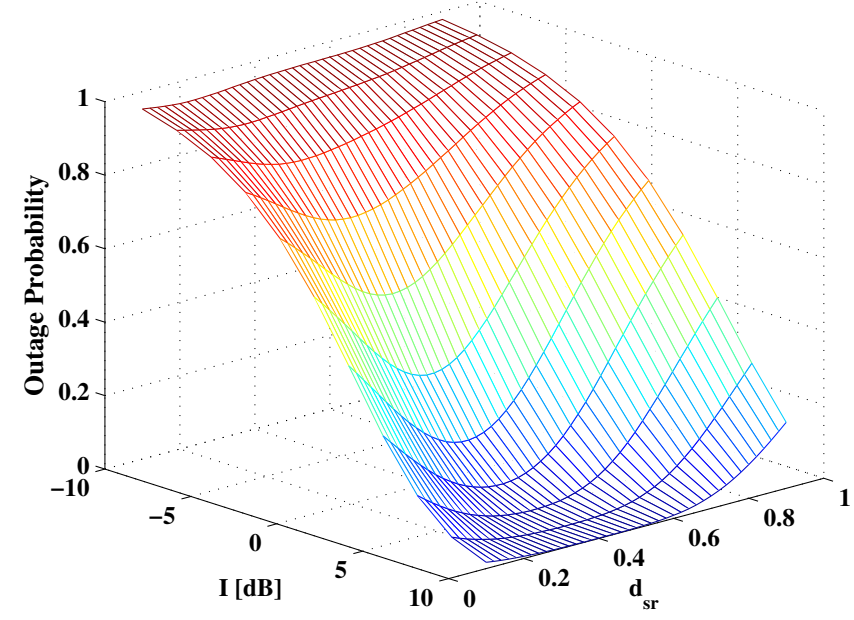

Figure 4. Outage Probability vs Interference threshold and $d_{\mathrm{sr}}$ for $\mathrm{R}=2$ and $\mathrm{K}=4$.

performance of the direct secondary link to that of the secondary system when assisted by a number of relays equals either to 4 or 8 .

\section{CONCLUSION}

In this contribution, an exact outage analysis of the relay-aided secondary link has been derived and validated through simulation results. Also, it has been shown that incorporating relays that act under the framework of half-duplex selective relaying leads to a substantial increase in the secondary system transmission reliability. Therefore, more degrees of freedom are given to the 


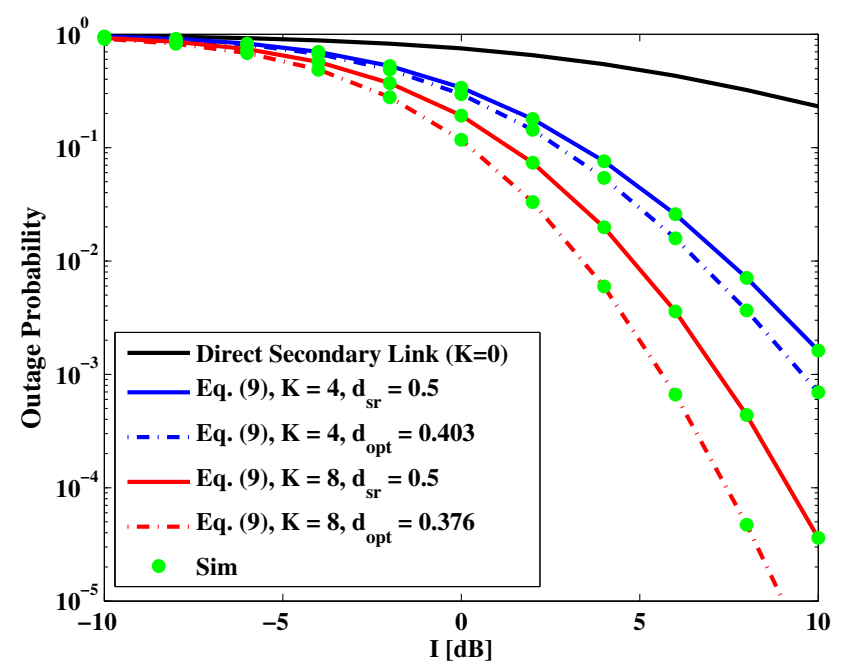

Figure 5. Outage Probability vs Interference threshold for $\mathrm{R}=2$.

secondary system to acquire a targeted QoS under the primary system interference constraint.

\section{APPENDIX}

Prior to deriving the second probability $P_{\text {out }}^{2}(\mathrm{I}, \Phi, \mathrm{i}, \mathrm{K})$, we begin by considering that a number of $i \in\{1, \ldots, \mathrm{K}\}$ relays from all the available $\mathrm{K}$ relays have succeeded to meet the threshold $\Phi$ during the first-hop and, without loss of generality, grouped within $C$ like $\left\{R_{1} \ldots, R_{\mathrm{i}-1}, R_{\mathrm{i}}\right\}$ (next, it is quiet remarkable that this prior consideration has no impact on the final derivation of $\left.P_{\text {out }}^{2}(\mathrm{I}, \Phi, \mathrm{i}, \mathrm{K})\right)$. Therefore, we can express $P_{\text {out }}^{2}(\mathrm{I}, \Phi, \mathrm{i}, \mathrm{K})$ after giving its definition in (12) as

$$
P_{\text {out }}^{2}(\mathrm{I}, \Phi, \mathrm{i}, \mathrm{K})=\int_{0}^{\Phi} P_{1}(y) f_{\gamma_{\mathrm{rs}|| C \mid=\mathrm{i}}}(y) d y,
$$

where $f_{\gamma_{\mathrm{rs}|| G \mid=\mathrm{i}}}$ corresponds to the probability distribution function (PDF) of $\gamma_{\mathrm{rs} \| G \mid=\mathrm{i}}$ (whose CDF is reported in (8)), while the conditional probability $P_{1}(y)$ is given by

$$
\begin{gathered}
P_{r}\left[\gamma_{\mathrm{ss}}<\Phi-y, \gamma_{\mathrm{s} 1} \geq \Phi, \ldots, \gamma_{\mathrm{si}} \geq \Phi, \gamma_{\mathrm{si}+1}<\Phi,\right. \\
\left.\ldots, \gamma_{\mathrm{sK}}<\Phi \mid y\right] .
\end{gathered}
$$

In turn, $P_{1}(y)$ may be expressed as.

$$
P_{1}(y)=\int_{0}^{+\infty} P_{2}(y, z) f_{\left|h_{\mathrm{sp}}\right|^{2}}(z) d z
$$

where $f_{\left|h_{\mathrm{sp}}\right|^{2}}(z)$ is the PDF of $\left|h_{\mathrm{sp}}\right|^{2}$ and $P_{2}(y, z)$, using the conditional CDFs (5) and (6) with respect to $z=\left|h_{\mathrm{sp}}\right|^{2}$, can be expressed as

$$
P_{2}(y, z)=F_{\gamma_{\mathrm{ss}} \mid z}(\Phi-y) \prod_{\mathrm{m}=1}^{\mathrm{i}}\left(1-F_{\gamma_{\mathrm{sm}} \mid z}(\Phi)\right) \prod_{\mathrm{n}=1}^{\mathrm{K}-\mathrm{i}} F_{\gamma_{\mathrm{sn}} \mid z}(\Phi) \text {. }
$$

Because of the independence between the two variables $\left|h_{\mathrm{sp}}\right|^{2}$ and $\left|h_{\mathrm{kp}}\right|^{2}$ for $\mathrm{k} \in\{1, \ldots, \mathrm{i}\}$, inverting the double integrals in (15) after replacing $P_{1}(y)$ by its expression given in (16), and applying the integration by parts to the second one will lead to the following formula

$$
P_{\text {out }}^{2}(\mathrm{I}, \Phi, \mathrm{i}, \mathrm{K})=\sum_{\mathrm{m}=0}^{\mathrm{K}-\mathrm{i}}\left(\begin{array}{c}
\mathrm{K}-\mathrm{i} \\
\mathrm{m}
\end{array}\right)(-1)^{\mathrm{m}}\left[\frac{F_{\gamma_{\mathrm{rs} \| C \mid=\mathrm{i}}}(\Phi)}{\frac{(\mathrm{i}+\mathrm{m}) \Phi \lambda_{\mathrm{sp}}}{\mathrm{I} \lambda_{\mathrm{sr}}}+1}-\mathcal{I}\right]
$$

where the integral $\mathcal{I}$ in (18) equals to

$$
\mathcal{I}=F_{\gamma_{\mathrm{rs}|| G \mid=\mathrm{i}}}(\Phi)-\sum_{\mathrm{n}=0}^{\mathrm{i}}\left(\begin{array}{c}
\mathrm{i} \\
\mathrm{n}
\end{array}\right)(-1)^{\mathrm{n}} \underbrace{\int_{0}^{\Phi} \frac{1}{(B-C y)^{2}(1+D y)^{\mathrm{n}}} d y}_{\mathcal{J}},
$$

and the conditional $\operatorname{CDF} F_{\gamma_{\mathrm{rs}|| G \mid=\mathrm{i}}}(\Phi)$ is given by (8). The integral $\mathcal{J}$ in (19) can be derived in its closed hypergeometrical form which can be checked on the wolfram mathematica online integrator to be given by,

$$
\mathcal{J}=-C^{\mathrm{n}}\left[\frac{{ }_{2} \mathrm{~F}_{1}\left(\mathrm{n}, \mathrm{n}+1, \mathrm{n}+2, \frac{C+B D}{D(B-C y)}\right)}{(\mathrm{n}+1) D^{\mathrm{n}}(C y-B)^{\mathrm{n}+1}}\right]_{0}^{\Phi}
$$

leading finally to (13).

\section{REFERENCES}

[1] A. Glodsmith, S. A. Jafar, I. Marić, and S. Srinivasa, "Breaking spectrum gridlock with cognitive radios: An information theoretic perspective," Proceedings of the IEEE, vol. 97, no. 5, pp. 894-914, May 2009.

[2] Y. Zou, J. Zhu, B. Zheng, and Y.-D. Yao, "An adaptive cooperation diversity scheme with best-relay selection in cognitive radio networks," IEEE Transactions Signal Processing, vol. 58, no. 10, pp. 5438 -5445, 2010.

[3] J. Lee, H.Wang, J. G. Andrews, and D. Hong, "Outage probability of cognitive relay networks with interference constraints," IEEE Transactions on Wireles Communications, vol. 10, no. 2, pp. 390-395, Feb. 2011.

[4] L. Luo, P. Zhang, G. Zhang, and J. Qin, "Outage performance for cognitive relay networks with underlay spectrum sharing," IEEE Communications Letters, vol. 15, no. 7, pp. 710-712, Jul. 2011.

[5] H. Ding, J. Ge, D. B. da Costa, and Z. Jiang, "Asymptotic analysis of cooperative diversity systems with relay selection in a spectrum-sharing scenario," IEEE Transactions on Vehicular Technology, vol. 60, no. 2, pp. 457-472, Feb. 2011.

[6] T. Q. Duong, V. N. Q. Bao, and H. J. Zepernick, "Exact outage probability of cognitive AF relaying with underlay spectrum sharing," Electronics Letters, vol. 47, no. 17, Aug. 2011.

[7] W. Jaafar, W. Ajib, and D. Haccoun, "A novel relay-aided transmission scheme in cognitive radio networks," in Proc. IEEE Global Communications Conference, Houston, Texas, USA, Dec. 2011.

[8] S. Ikki, M. Uysal, and M. H. Ahmed, "Performance analysis of incrementalbest-relay amplify-and-forward technique," in Proc. IEEE Global Communications Conference, Honolulu, Hawaii, USA, Dec. 2009.

[9] A. Bletsas, A. Khisti, D. P. Reed, and A. Lippman, "A simple cooperative diversity method based on network path selection," IEEE Journal on Selected Areas in Communications, vol. 24, no. 3, pp. 659-672, Mar. 2006.

[10] J. Si, Z. Li, X. Chen, B. Hao, and Z. Liu, "On the performance of cognitive relay networks under primary user's outage constraint," IEEE Communications Letters, vol. 15, no. 4, pp. 422-424, Apr. 2011.

[11] Z. Yan, X. Zhang, and W. Wang, "Exact Outage Performance of Cognitive Relay Networks with Maximum Transmit Power Limits," IEEE Communications Letters, vol. 15, no. 12, pp. 1317-1319, Dec. 2011. 